\title{
The Effects of Viewing Natural Scenery Indoors on Middle School Students' Changes in Mood States and Concentration
}

\author{
Mun-Young Shin ${ }^{1}$ and Won-Sop Shin ${ }^{2 *}$ \\ ${ }^{1}$ Graduate Department of Forest Therapy, Graduate School, Chungbuk National University, Cheongju 28644, Korea \\ ${ }^{2}$ Department of Forestry, Chungbuk National University, Cheongju 28644, Korea
}

\begin{abstract}
The purpose of this study was to investigate the mood states and concentration of students when they see the natural scenery indoors throughout the Free Semester Program (FSP) of middle school. The subjects were total 180 students comprised of 60 students in the group viewing natural scenery, 60 students in the group viewing urban scenery and 60 students in the control group from the first year of middle school located in Daejeon, and the experiment was carried out from September 3 to October 30, in 2018. The results showed that tension, depression, anger, fatigue and confusion were decreased and vigor was increased significantly in group viewing natural scenery compared to group viewing urban scenery and the control group. Ratings of 'pleasant,' 'natural,' and 'calm' were increased and concentration was also improved significantly in the group viewing natural scenery compared to the group viewing urban scenery and the control group. This indicates that positive effects can be anticipated on the improvement of mood states and concentration by developing indoor program viewing natural scenery for people who have difficulties in outdoor activities.
\end{abstract}

Keywords: concentration, mood, natural scenery effect, stress

\section{Introduction}

According to the 9th International Comparison of the Happiness Index for Korean Children and Adolescents 2017 surveyed by Korea Bang Jeong-hwan Foundation and Yonsei University Institute for Social Development Studies (Korea Bang Jeong-hwan Foundation, 2017) on adolescents in South Korea, the subjective happiness index of Korean adolescents was 87.6 (m-100) points. Korea was the lowest of the 22 member countries of the OECD, which indicates that happiness and psychological well-being of Korean adolescents are severely threatened. Meanwhile, according to the National Health Insurance Service (2014), the number of teenage adolescents that received treatment at neuropsychiatric hospitals due to stress-related disorders increased from 5,838 in 2004 to 36,782 in 2012, increased by at least six times in eight years. This result shows that many adolescents are suffering from a high level of stress, and their mental health is also threatened by it.

Adolescence is the time in which they undergo great changes and developmental crises physically, psychologically and

Received: February 8, 2019, Revised: February 26, 2019, Accepted: April 2, 2019

First author: Mun-Young Shin, E-mail: alang322@naver.com, (1) https://orcid.org/0000-0002-1525-4041

*Corresponding author: Won-Sop Shin, E-mail: shinwon@chungbuk.ac.kr, (1) https://orcid.org/0000-0002-5665-1502 
environmentally, thereby suffering stress. Adolescents in Korea particularly have a high level of stress about their studies and college admissions, thereby exposed to greater stress than other periods (Lee and Jang, 2011). A certain level of exam stress induces academic motivation and helps improve grades, but once it exceeds that level, it deprives students of confidence about stress, and deteriorates the will to study as well as behavioral efficiency. Students may even feel their heart beat faster, be sick to their stomach, feel impatient and hasty, become oversensitive to trivial things, and even experience depression neurosis in severe cases (Lee and Larson, 1996).

To solve such diverse problems of adolescents, the Ministry of Education has made the implementation and operation of the Free Semester Program (FSP) mandatory for all middle schools since 2016. The FSP enables middle school students to break free from education based on knowledge and competition, and participate in various beneficial experiences by offering all kinds of programs at school such as classes based on student participation and various activities. Recently there are a variety of FSPs using forest and natural resources such as forest activities at school or green job experiences, and these programs are known to have many positive effects both psychologically and physiologically.

Natural and green scenery is known to be reducing negative emotions such as fear, anger, aggression, tension and anxiety, while increasing positive emotions (Ulrich et al., 1991; Hartiga et al., 1991), and merely viewing natural scenery eliminates complicated thoughts and refreshes oneself, thereby removing mental fatigue and stress and inducing positive psychological state according to Yi and Yi (2006). Natural and green scenery makes people concentrate in the modern society where there are various external stimulations, and this concentration is not intentional and thus eliminates mental fatigue in daily life (Kaplan, 1995). Thus, human beings feel comfortable and pleasant in the forest that has less external stimulations and does not require as much attention unlike the urban environment. This attention restoration theory provides a framework suitable for analyzing the effect of viewing indoor scenery on students' mood swings and changes in concentration.

According to the statistics by Robert Muir, $85 \%$ of what we perceive from the outside is through vision, $10 \%$ through hearing, $4 \%$ through smell, $2 \%$ through touch, and $1 \%$ through taste (Cheon, 2010). As such, a positive example of exposure to natural scenery is recently presented media studies, reporting the effect of viewers' experience of being exposed to natural scenery on through a TV screen on the buffering of their negative emotions (Choi, 2014). On the contrary, there are negative changes such as chromosome breakage when unconsciously exposed to city noises for a long time (Dorado-Correa et al., 2018).

Many studies have been conducted in Korea and overseas using healing by natural scenery and changes in mood states. Even though there are many previous studies proving the effect of scenery such as the influence of forest and urban scenery on human sensibility (Eom, 2016), psychological or physiological effect of viewing forests and taking walks (Park, 2010; Ji et al., 2012), and effect of indirect forest experience on the psychology of human body (Jeon, 2017), not enough studies used direct viewing of landscapes indoors in the program. Moreover, insufficient case studies have been done on middle school students compared to forest therapy case studies on adults, young children or elementary school students.

Stimulation of forests turned out to have psychologically and physiologically positive effects just like field experience even when done indirectly indoors (Jeon, 2017). Based on these results, it is necessary to provide simple and effective programs indoors within the required time frame for middle school students who cannot frequently use outdoor programs.

This study is to provide a simple forest therapy program for students who concentrate on studying all day to relax for a moment by viewing natural scenery indoors with the FSP, thereby giving the effect of forest therapy for middle school students who do not have much free time for outdoor activities, and verify the effects of viewing indoor natural scenery on their mood swings and concentration. 
The national park lecture-type green job program of the FSP is used to come up with a single program that added viewing of scenery to the green job program. The results were measured for the group that viewed natural scenery, group that viewed urban scenery, and group that did not view any scenery to determine changes in mood states and concentration.

\section{Research Methods}

\section{Subjects}

This study was conducted on students in the first year of middle school among those that applied for the national park green job program of the FSP and that is carrying out the Free Semester classes in Daejeon. The national park green job program enables middle school students, who are the future generation, to experience various green jobs at the national park with ecologically excellent natural environment and expertise for their sustainable use and growth, and promotes their understanding about the duties of the national park and provide career exploration opportunities. This provides a chance for them to think about their career by having a new experience in nature away from the pressure of their studies, giving them opportunities to cultivate environmental sensitivity and have environmental education. It was conducted from September 3 to October 30, 2018 on total 180 subjects (90 male, 90 female), divided into a group of 60 subjects (30 male, 30 female) viewing natural scenery, a group of 60 subjects (30 male, 30 female) viewing urban scenery, and a group of 60 subjects ( 30 male, 30 female) not viewing scenery. The classroom environment and conditions for textbooks and teaching materials were set up equally for all three groups.

For the experimental groups, the process of experiment was explained to the teacher in charge of the FSP's career experience and the students, and their consent was also obtained before the program.

\section{Implementation method}

According to Kaplan's attention restoration theory (ART), the forest restores the human attention, which had not been focused due to various complicated factors of the surrounding environment. Excessive attentional fatigue caused by external environmental factors or internal psychological factors is recovered by the restorative environment of the forest, making people feel comfortable and pleasant. Thus, this study assumes that giving time for middle school students who cannot encounter nature all day to encounter the colors and sounds of nature for even a brief time will help them relax their stiff body and mind, thereby restoring attention and significantly affecting positive emotions and concentration.

Visual media of natural scenery was created on PowerPoint by adding the sounds of nature to photographs of natural scenery.

The visual images are comprised of photographic works that won awards at the photo exhibitions of national parks. White noises such as the sounds of water, birds and winds in nature were added to the beautiful landscapes of nature, forest paths, valleys, lakes and waterfalls, which were arranged to maximize the emotions that can be actually felt in the mountains even through an indirect experience. The biggest feature of the sounds of nature is the energy throughout the entire frequency band. Sounds of rain or waterfall include such character of full band frequency, having similar effects as white noises such as promoting concentration and blocking ambient noise (Kyon and Bae, 2009). The forest sounds are about 40-50 dB (Park and Kim, 2010), which is the level that is most relaxing to hear by humans. The level was set to 40-50 dB when adjusting the volume of the visual images. Scenes in which people appear were eliminated to maintain the engagement of the participants. Total 20 photographs were shown for 10 minutes, 30 seconds per photograph, considering that the subjects are first-year middle school students. For the sounds of the forest and city, sounds that were actually recorded and 
white noises were mixed and edited using Sound Forge 11.0 and Goldwave v6.31.

For the visual images of urban scenery, artificial elements such as buildings, roads and cars were arranged, adding sounds of cars, winds and horns in the city to maximize the emotions felt directly in the city even through an indirect experience. The sound level for urban scenery was also set to $40-50 \mathrm{~dB}$, which is the level that is most relaxing to hear by humans. The time of showing the visual images or urban scenery was also set equally as the experimental group, showing 20 photographs for 10 minutes, 30 seconds per photograph, considering that the subjects are first-year middle school students.

Both forest scenery group and urban scenery group participated in the national park FSP green job program and after that, viewed natural scenery and urban scenery, respectively. Control group only participated in the national park FSP green job program.

\section{Methods and tools of experiment}

\section{Profile of Mood States (POMS)}

The POMS was developed in 1964 due to the clinical need to quickly and simply determine the temporary and changeable affects of humans, after which it has been widely used to measure the mood states and emotions of humans. This study used the Korea Version of Profile of Mood States-Brief (KPOMS-B) adapted by Yeun and Shin-Park (2006).

Total 30 items were rated on a 5-point scale, comprised of 6 categories such as tension, depression, anger, vigor, fatigue and confusion (total score of 25 per category). The scores of each category are added up to evaluate total mood disturbance (TMD; McNair and Lorr, 1964).

\section{Semantic Differential (SD) method questionnaire}

The SD is to measure the human space of image with adjectives expressing human emotions, and was developed by Osgood (1964) for cross-cultural studies investigating how similar the meanings of vocabularies in different countries of the world are. This has been widely used in landscape evaluation including sensibility engineering or appraisal and assessment, and this study conducted a survey using three adjectives of 'pleasant-unpleasant,' 'natural-artificial,' and 'excitedcalm' rated on a scale of 13 , using the opposite pairs of adjectives after stimulation in the assessment of mood states.

\section{Measurement of concentration (concentration grid)}

To measure whether the scenery viewing program has effect on increasing concentration, this study used the concentration grid by Harris and Harris (1984). The standard is a $10 \times 10$ grid on square paper of $25 \mathrm{~cm} \times 25 \mathrm{~cm}$, arranging numbers from zero to 99 on the boxes. The students are to find the numbers starting from 20 in the pretest measurement and from 60 in the posttest measurement within 60 seconds. Students place the paper on their desks and look straight ahead, and then find the numbers that come after the ones indicated by the researcher and mark the relevant numbers. The numbers found are counted as 1 point per number, and students that found more numbers have higher level of concentration.

\section{Data analysis}

This study used the following method and procedures of analysis to solve the research question and test the hypothesis. SPSS 18.0 program was used for analysis.

To determine the POMS of the group that viewed natural scenery indoors, the group that viewed urban scenery, and the 
group that did not view any scenery as well as the SD and concentration difference among stimulations according to elements, this study examined the mean and standard deviation and conducted a one-way ANOVA. The significance level was $p<.05$.

\section{Results and Discussion}

\section{Result of homogeneity test among groups}

As a result of testing homogeneity among groups before applying the indoor scenery viewing program, the groups were proved to be statistically homogeneous for the sub-factors of mood states (tension, depression, anger, fatigue, confusion, vigor) and concentration as shown in Table $1(p>.05)$.

\section{Changes in mood states}

One-way ANOVA was conducted to verify whether there is a significant difference in mood states according to scenery indoors. The result showed that there was a significant difference in tension $(\mathrm{F}=17.82, p<.001)$, depression $(\mathrm{F}=23.13$, $p<.001)$, anger $(\mathrm{F}=19.39, p<.001)$, fatigue $(\mathrm{F}=17.53, p<.001)$, and confusion $(\mathrm{F}=17.04, p<.001)$ according to scenery, and there was also a statistically significant result in vigor $(\mathrm{F}=18.16, p<.001)$ (Table 2$)$. The group viewing natural scenery indoors showed positive changes and a decrease in negative emotions after the program compared to the group viewing urban scenery and the group not viewing any scenery. The result of data analysis showed that there is a significant result in all sub-factors of mood states among the groups. The result was similar to that of direct experience in which viewing forest scenery decreased tension, anxiety, anger, fatigue and confusion and increased vigor compared to viewing urban scenery, while also decreasing TMD (Park, 2010).

\section{Semantic Differential (SD) method}

Semantic Differential, which expresses the imagery of the stimulation from scenery, shows the results as presented in Table 3 comparing pleasant, natural and calm among the group viewing natural scenery, the group viewing urban scenery and the group not viewing any scenery. The sense of feeling pleasant $(\mathrm{F}=22.56, p<.001)$, natural $(\mathrm{F}=29.73, p<.001)$ and calm $(\mathrm{F}=23.47, p<.001)$, by the group viewing natural scenery that received comprehensive stimulation of the forest indoors increased more significantly than the group viewing urban scenery that received comprehensive stimulation of

Table 1. Result of homogeneity among groups

\begin{tabular}{llcccc}
\hline Item & & Levene statistic & df1 & df2 & $p$ \\
\hline \multirow{4}{*}{ Profile of } & Tension & 1.781 & 2 & 177 & .171 \\
Mood States & Depression & .857 & 2 & 177 & .426 \\
& Anger & 1.904 & 2 & 177 & .152 \\
& Fatigue & .126 & 2 & 177 & .882 \\
& Confusion & .007 & 2 & 177 & .993 \\
& Vigor & .811 & 2 & 177 & .446 \\
\hline Concentration & & 2.305 & 2 & 177 & .103 \\
\hline
\end{tabular}


Table 2. Comparison of pre- and post-test in the Profile of Mood States (POMS) among groups

\begin{tabular}{|c|c|c|c|c|c|c|c|}
\hline \multirow{2}{*}{ Item } & \multirow{2}{*}{ Time } & \multicolumn{3}{|c|}{$\mathrm{M} \pm \mathrm{SD}$} & \multirow{2}{*}{$\mathrm{F}$} & \multirow{2}{*}{$p$} & \multirow{2}{*}{ Scheffe $^{\mathrm{z}}$} \\
\hline & & Forest scenery group & Urban scenery group & Control group & & & \\
\hline \multirow{2}{*}{ Tension } & Pre & $7.76 \pm 3.67$ & $7.66 \pm 3.21$ & $7.78 \pm 3.52$ & \multirow{2}{*}{17.82} & \multirow{2}{*}{$.000^{* * * *}$} & \multirow{2}{*}{$\mathrm{a}<\mathrm{c}<\mathrm{b}$} \\
\hline & Post & $6.45 \pm 1.90$ & $9.66 \pm 4.12$ & $7.45 \pm 2.59$ & & & \\
\hline \multirow{2}{*}{ Depression } & Pre & $8.10 \pm 3.19$ & $8.18 \pm 2.94$ & $8.11 \pm 3.07$ & \multirow{2}{*}{23.13} & \multirow{2}{*}{$.000^{* * *}$} & \multirow{2}{*}{$\mathrm{a}<\mathrm{c}<\mathrm{b}$} \\
\hline & Post & $6.60 \pm 2.65$ & $10.23 \pm 3.78$ & $7.80 \pm 2.34$ & & & \\
\hline \multirow{2}{*}{ Anger } & Pre & $8.08 \pm 3.96$ & $8.01 \pm 3.12$ & $8.03 \pm 3.61$ & \multirow{2}{*}{19.39} & \multirow{2}{*}{$.000^{* * * *}$} & \multirow{2}{*}{$\mathrm{a}<\mathrm{c}<\mathrm{b}$} \\
\hline & Post & $6.56 \pm 2.43$ & $10.13 \pm 4.36$ & $7.80 \pm 2.34$ & & & \\
\hline \multirow{2}{*}{ Fatigue } & Pre & $9.36 \pm 3.83$ & $9.35 \pm 3.46$ & $9.33 \pm 3.63$ & \multirow{2}{*}{17.53} & \multirow{2}{*}{$.000^{* * *}$} & \multirow{2}{*}{$\mathrm{a}<\mathrm{c}<\mathrm{b}$} \\
\hline & Post & $7.53 \pm 3.35$ & $11.15 \pm 4.02$ & $8.98 \pm 2.57$ & & & \\
\hline \multirow{2}{*}{ Confusion } & Pre & $9.03 \pm 3.45$ & $9.06 \pm 3.43$ & $9.08 \pm 3.43$ & \multirow{2}{*}{17.04} & \multirow{2}{*}{$.000^{* * * *}$} & \multirow{2}{*}{$\mathrm{a}<\mathrm{c}<\mathrm{b}$} \\
\hline & Post & $7.55 \pm 2.94$ & $10.65 \pm 3.13$ & $8.80 \pm 2.67$ & & & \\
\hline \multirow{2}{*}{ Vigor } & Pre & $12.21 \pm 5.42$ & $12.20 \pm 4.75$ & $12.03 \pm 5.60$ & \multirow{2}{*}{18.16} & \multirow{2}{*}{$.000^{* * *}$} & \multirow{2}{*}{$\mathrm{b}<\mathrm{c}<\mathrm{a}$} \\
\hline & Post & $14.65 \pm 5.30$ & $10.20 \pm 3.61$ & $12.76 \pm 2.87$ & & & \\
\hline \multirow{2}{*}{$\mathrm{TMD}^{\mathrm{y}}$} & Pre & $30.33 \pm 16.06$ & $30.08 \pm 13.78$ & $30.28 \pm 15.21$ & \multirow{2}{*}{33.90} & \multirow{2}{*}{$.000^{* * *}$} & \multirow{2}{*}{$\mathrm{a}<\mathrm{c}<\mathrm{b}$} \\
\hline & Post & $20.43 \pm 13.10$ & $41.63 \pm 16.74$ & $28.95 \pm 12.33$ & & & \\
\hline
\end{tabular}

$\mathrm{z}_{\mathrm{a}}=$ forest scenery group $\mathrm{b}=$ urban scenery group; $\mathrm{c}=$ control group.

${ }^{\mathrm{y}} \mathrm{TMD}=$ Total Mood Disturbance.

${ }^{*} p<.05, \stackrel{* *}{p}<.01,{ }^{* * *} p<.001$.

Table 3. Comparison of post-test in the Semantic Differential (SD) method among groups

\begin{tabular}{|c|c|c|c|c|c|c|}
\hline \multirow{2}{*}{ Item } & \multicolumn{3}{|c|}{$\mathrm{M} \pm \mathrm{SD}$} & \multirow{2}{*}{$\mathrm{F}$} & \multirow{2}{*}{$p$} & \multirow{2}{*}{ Scheffe } \\
\hline & Forest scenery group & Urban scenery group & Control group & & & \\
\hline Pleasant & $3.77 \pm 1.99$ & $0.85 \pm 3.23$ & $1.85 \pm 1.75$ & 25.56 & $.000^{* * *}$ & $\mathrm{~b}<\mathrm{c}<\mathrm{a}$ \\
\hline Natural & $3.90 \pm 2.22$ & $0.43 \pm 3.28$ & $1.98 \pm 1.58$ & 29.73 & $.000^{* * *}$ & $\mathrm{~b}<\mathrm{c}<\mathrm{a}$ \\
\hline Calm & $4.05 \pm 2.19$ & $1.12 \pm 3.26$ & $1.77 \pm 1.65$ & 23.47 & $.000^{* * * *}$ & $\mathrm{~b}, \mathrm{c}<\mathrm{a}$ \\
\hline
\end{tabular}

${ }^{2} \mathrm{a}=$ forest scenery group; $\mathrm{b}=$ urban scenery group; $\mathrm{c}=$ control group.

${ }^{*} p<.05, \stackrel{* *}{p} p<.01,{ }^{* * *} p<.001$.

urban scenery and the group not viewing any scenery. Other studies also reported similar results that the forest environment induces positive emotions, increases happiness, and decreases aggression, anger and stress level (van den Berg et al., 2003; Hartig et al., 2003; Wells and Evans, 2003).

\section{Changes in concentration}

As for the difference in concentration according to viewing of scenery indoors, concentration was more significant in the group viewing natural scenery $(\mathrm{F}=23.77, p<.01)$ than the two other groups (Table 4$)$. This is similar to other studies which proved the improvement of happiness after watching scenes of natural landscape (Choi and Moon, 2016), and which claimed that the sounds of traffic in the city create remarkably more beta waves than the sounds of nature, while the sounds of nature create more alpha waves on the contrary (Kyon and Bae, 2009). 
Table 4. Comparison of pre- and post-test in the measurement of concentration among groups

\begin{tabular}{|c|c|c|c|c|c|c|}
\hline \multirow{2}{*}{ Item } & \multicolumn{3}{|c|}{$\mathrm{M} \pm \mathrm{SD}$} & \multirow{2}{*}{$\mathrm{F}$} & \multirow{2}{*}{$p$} & \multirow{2}{*}{ Scheffe ${ }^{\mathrm{z}}$} \\
\hline & Forest scenery group & Urban scenery group & Control group & & & \\
\hline Pre & $6.53 \pm 2.61$ & $6.73 \pm 2.09$ & $6.58 \pm 2.00$ & \multirow{2}{*}{23.77} & \multirow{2}{*}{$.002^{* *}$} & \multirow{2}{*}{$b, c<a$} \\
\hline Post & $9.08 \pm 3.56$ & $5.85 \pm 1.08$ & $6.93 \pm 2.05$ & & & \\
\hline
\end{tabular}

${ }_{\mathrm{z}} \mathrm{a}=$ forest scenery group; $\mathrm{b}=$ urban scenery group; $\mathrm{c}=$ control group.

${ }^{*} p<.05, \stackrel{* *}{p}<.01,{ }^{* * *} p<.001$.

\section{Conclusion}

This study is conducted to determine the effects of viewing natural scenery indoors in the middle school FSP on the mood states, imagery and concentration of middle school students. The objective is in seeking ways that make forest therapy constantly possible indoors based on the positive results of viewing natural scenery indoors. The experiment was conducted on students in the first year of middle school to verify the effectiveness on mood states and concentration when participating in the natural scenery program indoors instead of in the forest therapy program outdoors through the FSP class. It was conducted on total 180 students comprised of 60 students in the group viewing indoor natural scenery, 60 students in the group viewing urban scenery, and 60 students in the group not viewing any scenery. Surveys were conducted before and after the program to determine the effects on mood swings and concentration.

The results showed that the group viewing natural scenery showed a decrease in tension, anger, depression, fatigue and confusion and a significant increase in vigor compared to the group viewing urban scenery and the group not viewing any scenery. Furthermore, the sense of feeling pleasant, natural and calm also increased significantly when the students received complex stimulation of the forest indoors, and concentration also increased significantly in the group viewing natural scenery compared to the other two groups. These results support the findings of previous studies that contact with nature has positive effects on health and happiness (Mackerron and Mourato, 2013), and merely watching a video of preferred natural scenery such as the ocean or forest can relieve tension and reduce fatigue, thereby obtaining the relaxing effect of the forest without having to physically visit the forest (Mortia et al., 2007). Moreover, the experience of viewing natural scenery on screen reduced depression or anxiety in terms of emotional effect, while increasing positive mood (Choi and Moon, 2016), and the sounds of nature refresh the mood and increase concentration, showing the effect of mental stability (Park, 2005). This indicates that the program viewing natural scenery indoors helps improve mood states and concentration of students.

A few suggestions can be made for follow-up research based on the conclusions.

First, the effect of the natural scenery viewing program is based on short-term experience and thus has difficulty in generalizing the result with expanded scope. Thus, it is necessary to verify the emotional effects of viewing natural scenery through constant research that is more long-term and systematic, expanding the scope of methods such as by school year, gender and school district.

Second, this study is to verify the effectiveness of the indoor forest therapy program and provide the effect of forest experience for those who cannot actually visit the forest. It is necessary to develop various indoor forest therapy programs to bring in the diverse natural resources of the forest in various ways to produce psychologically and physiologically positive effects.

Therefore, this study is expected to contribute to developing more specialized, systematic and diverse programs beyond the limitations of a forest therapy program for people who have difficulty in accessing the natural environment due to 
mobility difficulties or dementia, or those who cannot actually visit and experience the forest directly due to sudden environmental factors such as particulate matter or rain.

\section{References}

Cheon, M.S. 2010. A study on the five senses and interactivity of brand design. J. Brand Des. Assoc. Korea. 8(1):190-199.

Choi, Y.J. 2014. Effects of nature scenery in TV screen on the viewers' stress buffering. Soc. Sci. Res. Inst. [Kyungsung University] 30(4):523-547.

Choi, Y.J. and S.J. Moon. 2016. The effect of natural scenery from TV media on viewers' happiness. Tour. Manag. 31(2): 407-425.

Dorado-Correa, A.M., S.A. Zollinger, B. Heidinger, and H. Brumm. 2018. Timing matters: Traffic noise accelerates telomere loss rate differently across developmental stages. Front. Zool. 15:29. https://doi.org/10.1186/s12983-018-0275-8

Eom, P.D. 2016. Effect of forestscape and cityscape on human emotion. Doctoral dissertation, Sangmyung University, Seoul, Korea.

Harris, D.V. and B.L. Harris. 1984. The athlete's guide to sports psychology: Mental skills for physical people. New York, NY: Leisure Press.

Hartiga, T. and G.W. Evans, L.D. Jamnerc, D.S. Davis, and T. Garling. 2003. Tracking restoration in natural and urban field settings. J. Environ. Psychol. 23(2):109-123. https://doi.org/10.1016/S0272-4944(02)00109-3

Hartiga, T.A., A. Mang and G.W. Evans. 1991. Restorative effects of natural environment experience. Environ. Behav. 23(1):3-26. https://doi.org/10.1177/0013916591231001

Jeon, J.Y. 2017. The effects of direct and indirect forest experience on human psychology and physiology. Master's thesis, Chungbuk National University, Cheongju, Korea.

Ji, G.B., K.N. Kim, and G.S. Han. 2012. Physiological and psychological effects of viewing and walking in forest and urban area. J. Environ. Sci. Int. 21(5):605-611. https://doi.org/10.5322/JES.2012.21.5.605

Kaplan, S. 1995. The restorative benefits of nature : Toward an integrative framework. J. Environ. Psychol. 15(3):169-182. https://doi.org/10.1016/0272-4944(95)90001-2

Korea Bang Jeong-hwan Foundation. 2017, May 2. 2017 9th International research report on children and adolescents' happiness index in South Korea. Retrieved from http://www.korsofa.org

Kyon, D.H. and M.J. Bae. 2009. Analysis of acoustic psychology of city traffic and nature sounds. J. Acoust. Soc. Korea 28(4):356-362.

Lee, M. and R. Larson. 1996. Effectiveness if coping in adolescence: The case of Korean examination stress. Int. J. Behav. Dev. 19(4):851-869. https://doi.org/10.1177/016502549601900410

Lee, S.W. and Y.E. Jang. 2011. A study on the effect of adolescent's academic stress to suicidal ideation: Moderating effect of family cohesion. Korean J. Youth Stud. 18(11):111-136.

MacKerron, G. and S. Mourato. 2013. Happiness is greater in natural environments. Glob. Environ. Change 23(5):992-1000. https://doi.org/10.1016/j.gloenvcha.2013.03.010

McNair, D.M. and M. Lorr. 1964. An analysis of mood in neurotics. J. Abnorm. Soc. Psychol. 69(6):620-627. https://doi.org/10.1037/h0040902

Morita, E., S. Fukuda, J. Nagano, N. Hamajima, H. Yamamoto, Y. Iwai, T. Nakashima, H. Ohira, and T. Shirakawa. 2007. Psychological effects of forest environments on healthy adults: Shinrin-yoku (forest-air bathing, walking) as a possible method of stress reduction. Public Health. 121(1):54-63.

National Health Insurance Service. 2014. Retrieved from http://www.nhis.or.kr

Osgood, C.E. 1964. Semantic differential technique in the comparative study of cultures. Am. Anthropol. 66(3):171-200.

Park, B.J. 2010. Experimental approach of therapeutic effect of forest recreation activities: Focused on viewing and walking in forest environments. Doctoral dissertation, Choongnam National University, Daejeon, Korea. 
Park, J.H. 2005. The effects of meditation music with nature sound on primary school children's state anxiety. Master's thesis, Changwon National University, Changwon, Korea.

Park, M.H. and J.S. Kim. 2010, July 15. The healing power of forest ecosystem sound. Gangwondomin Daily News. Retrieved from http://www.kado.net/news/articleView.html?idxno=472871

Ulrich, R.S., R.F. Simons, B.D. Losito, E. Fiotito, M.A. Miles, and M. Zelson. 1991. Stress recovery during exposure to natural and urban environments. J. Environ. Psychol. 11(3):201-230. https://doi.org/10.1016/S0272-4944(05)80184-7

van den Berg, A.E., S.L. Koole, and N.Y. van der Wulp. 2003. Environmental preference and restoration: (How) are they related? J. Environ. Psychol. 23(2):135-146. https://doi.org/10.1016/S0272-4944(02)00111-1

Wells, N. M. and G. W. Evans. 2003. Nearby nature: A buffer of life stress among rural children. Environ. Behav. 35(3): 311-330.

Yeun, E.J. and K.K. Shin-Park. 2006. Verification of the profile of mood states-brief: Cross-cultural analysis. J. Clin. Psychol. 62(9):1173-1180. https://doi.org/10.1002/jclp.20269

Yi, Y.K. and P.I. YI. 2006. The impact of landscape type on urban office worker's stress and cognitive performance - Comparison between natural and urban landscape -. J. Korean Inst. Landsc. Archit. 33(6):1-11. 